\title{
3. Ärzte und Pflege: Feindliche Geschwister, oder der Weg zur Zusammenarbeit!
}

\author{
Uwe Kropp
}

\begin{abstract}
Fallbeispiel
Der Start in ein neues Arbeitsgebiet ist immer spannend. Frisch von der Uni oder bereits erfahren in anderen Fachgebieten. Egal, an welchem Ort man ankommt, es sind schon welche da. Mitarbeitende aller Berufsgruppen erwarten den Neuen oder die Neue.

Die Kolleginnen und Kollegen aus der eigenen Berufsgruppe erhoffen sich zusätzliche Hilfe und Unterstützung. Endlich werden die Dienste auf mehrere Schultern verteilt und die Arbeitsteilung kann zur Entspannung beitragen.

Alle könnten nun glücklich und zufrieden sein.

Nehmen wir die Sicht einer Pflegeperson ein, die nun schon über einige Jahre Berufserfahrung verfügt.

Diese Person kennt alle Abläufe und die Mitarbeiterinnen und Mitarbeiter. Sie ist praktisch im Unternehmen „groß“ geworden. Die Berufserfahrung bringt Sicherheit im Arbeitsalltag, denn sie hat ja schon so Einiges erlebt. Seit Jahren arbeitet sie auf der gleichen Station und hat schon viele Ärzte kommen und gehen sehen. Ärzte, die auch gerade zu Beginn, Fehler machen.

Nun also kommt der oder die Neue! Was für ein Typ Mensch wird er bzw. sie sein? Passt er ins Team? Immerhin wurde der neue Arzt bereits vom ärztlichen Leiter angepriesen! „Passt sehr gut ins Anforderungsprofil!“

$\mathrm{Hm}$, das hatten wir beim Letzten auch schon. „Na, warten wir mal ab!“

Der Tag der Wahrheit naht. Beide Berufsgruppen treffen im ersten Dienst natürlich aufeinander. Die Pflegeperson hat ja im Vorfeld bereits das eine oder andere gehört. „Keine Ahnung von Psychiatrie! Hat zuvor Neuro gemacht!“ „Denkt nun, die hierarchischen Strukturen der Somatik könnten hier auch gelten ..." „Ständig neue Anordnungen. Was soll das denn?“ „Kann wohl auch gut mit der Kollegin, die gerade ihre Fachweiterbildung abgeschlossen hat. Wollen neue Abläufe einführen und uns dann machen lassen.“ „Na, den lass mal kommen ....
\end{abstract}


Und ohne, dass beide viele Worte gewechselt haben, passiert was passieren muss: Die Begrüßung fällt frostig aus. Keine Chance - erst einmal.

Die einfache ärztliche Anordnung wird als Zumutung bewertet und ein Gespräch über einen Patienten wird mit den Sätzen beendet: „Den kenne ich seit Jahren, das bringt nix.“

Und nun zur Sicht des Arztes:

Neu und unerfahren im Fach Psychiatrie. Dafür aber die Erfahrung aus dem vorherigen Einsatz und ein gutes Verhältnis zu den Pflegekräften der Neuro gehabt. Lief immer sehr gut. Ja, so die eine oder andere Pflegeperson war schon mal zickig, aber letztendlich liefen die Anordnungen gut ab. Hat nie ganz verstanden, was die Pflegepersonen wollten, wenn es um das Ablegen der Befunde ging. War doch selbst kein Sekretär.

$\mathrm{Na}$ ja, wird mit den Psych-Schwestern schon genauso laufen. Hat ja auch nicht wirklich viel mit denen zu tun. Die ärztlichen Kolleginnen und Kollegen sind ja viel wichtiger. Vor der einen oder anderen Schwester wurde schon gewarnt.

So, oder so ähnlich könnte es passieren.

Welche Möglichkeit haben wir, dass Verständnis gegenseitig zu fördern und damit den Arbeitsalltag zu gestalten?

Hierfür bedarf es eines Erklärungsversuches zum jeweiligen Rollenverständnis der Berufsgruppe Ärzte und Pflege, aber auch, und dies ist entscheidend, eines Blickes möglicher „Aus-“Wirkung auf den Patienten.

Erklärungsversuch: Die traditionelle pflegerische Ausbildung der meisten erfahrenen Mitarbeiter des Pflegedienstes trägt noch den Stempel der medizinisch geprägten Ausbildungsgänge und war dementsprechend auch mit dem entsprechenden Rollenverständnis Arzt/Pflege verbunden. Die hierarchischen Strukturen der Krankenhäuser unterstützen oftmals die säulenhafte Trennung zwischen Arzt und Pflege.

Ob gewollt oder ungewollt, entstanden somit oft Abgrenzungen, die nicht fachlich zu begründen waren, sondern die sich auf einer subtilen emotionalen Ebene entwickelten.

Sicherlich waren die pflegerischen Ausbildungen an psychiatrischen Fachkrankenhäusern schon etwas differenzierter in der Rollenbetrachtung. Aber auch dort war der verantwortliche Leiter der Schule in aller Regel ein Arzt.

Der Pflegedienst musste sich daher schon immer irgendwie einen Platz „erkämpfen“.

Während sich im somatischen Bereich der Pflegedienst über zuvor sehr medizinisch orientierte Themen weiterentwickelte und Zusatzqualifikationen entweder das medizinischen Hintergrundwissen, wie z.B. in der Intensivmedizin, oder das Wissen über bestimmte Untersuchungs- und Behandlungstechniken (OP und Funktionsdiagnostik) vertieften, nahm die psychiatrische Krankenpflege schon in den 7oer-Jahren mit der Psychiatrie-Enquete einen enormen Veränderungsprozess auf. Durch die sich etablierende Sozialpsychiatrie nahm der Pflegedienst einen festen Platz im therapeutischen Team ein und erlangte Selbstbewusstsein und Vertrauen.

In den goer-Jahren brachte die Psychiatrie-Personalverordnung nochmals eine „Stärkung der Rolle“, da auch Aufgaben und somit Kompetenzen festgeschrieben wurden. Parallel zu dieser Entwicklung etablierten sich schon Ende der Goer- 
Jahre die ersten Fachweiterbildungen, die sich über die Jahre weiterentwickelten und nun perspektivisch von den ersten Studiengängen Psychiatrische Pflege abgelöst werden.

Pflegetheorien-Modelle, oftmals aus dem angloamerikanischen Sprachraum, erreichten die somatische und psychiatrische Pflege und somit war der Weg zur eigenständigen Profession, weg von dem Heil- und Hilfsberufsgedanken, beschritten.

Es ist ein Konfliktfeld zwischen Arzt und Pflege, welches bis in die Terminologie bestimmter Begriffe wie Pflegediagnose und Pflegevisite hineinzog.

Dass diese Entwicklung in einigen Bereichen Blüten der Abgrenzung trieb, die aus heutiger Sicht nicht zur Teambildung beitrugen, muss selbstkritisch zugegeben werden.

Sehr früh wurden im Pflegedienst Managementausbildungen etabliert, die ihrerseits Psychologie, Kommunikation, Personalführung und -entwicklung im Fokus hatten und somit auch dazu führten, dass sich der Pflegedienst gut gerüstet aufstellte und „Führung“, auch im Team, übernahm.

Demgegenüber das Studium der Humanmedizin. Jeder Medizinstudent lernt oder lernte in den klassischen Rollen und den in der Medizin stark verankerten Hierarchien. Erst neue Studiengänge der letzten Jahre brachten die Kommunikation mit in das Vorlesungsverzeichnis. Jedoch findet man selbst in den Modellstudiengängen am Beispiel Berlin, wie im Fach „KIT“ Kommunikation, Interaktion und Teamarbeit, kaum etwas zur wirklichen Zusammenarbeit zwischen den Berufsgruppen. Selbst das im Handbuch für Studierende im 4. Semester des Modellstudiengangs Medizin der Charité Berlin verwendete Beispiel für Probleme im Team bezog sich auf die Rollen Student/Assistenzarzt/Stationsarzt.

Somit bleibt auch die Arztrolle in der klassisch besetzten und von Parsons 1951 (Rothgangel 2010) formulierten Erwartungsrolle, die sich zwar auf das Arzt/Patientenverhältnis bezieht, aber nicht auf ein soziales System wie ein multiprofessionelles Team übertragen lässt:

- fachliche Kompetenz

- allparteiliche Haltung

- Respektierung der Beschränkung auf den gestellten Auftrag

- affektive Neutralität

- auf das Wohl der Gemeinschaft ausgerichtete Haltung

Ein solches Lernen bringt es zwangsläufig mit sich, dass der Blick des Arztes die Arzt/Patientensituation im Fokus hat und nicht unbedingt die anderen Akteure im Gesundheitswesen. Die in den letzten Jahren zunehmende Ökonomisierung bringt weiteren Druck in die Interaktionen.

Wenngleich sich auch im ärztlichen Dienst in den vergangenen Jahren zunehmend Managementausbildungen etablierten, die Personalführung, Personalentwicklung und Teamkommunikation mitbetrachten, bleibt doch zumindest auf dem Ausbildungsweg des jungen Mediziners das tradierte Rollenverständnis als gelebt und erlebt haften. 
- Jeder Beruf bringt sein Rollenverständnis ein und hat seine Geschichte.

- Kommunikation untereinander muss und kann gelernt werden. Sie basiert jedoch auf gegenseitigem Respekt, Wertschätzung und Anerkennung der Person. Lassen Sie sich als Arzt die psychiatrische Pflege doch einmal erklären!

\section{Vom Individuum zum Team und raus aus dem unnötigen Konfliktfeld!}

Das psychiatrische Arbeitsfeld ist geprägt von Kommunikation. Ärztliche Entscheidungen, die letztendlich in Anordnungen münden, basieren in aller Regel auf Informationen zum Patienten, die von unterschiedlichen Berufsgruppen zusammengetragen wurden. Umgekehrt laufen pflegerische Entscheidungsprozesse nicht ohne Abstimmung innerhalb des Gesamttherapieplanes. Die Arbeit im Team basiert daher auf der Kommunikation und der daraus ableitbaren $\mathrm{Zu}$ sammenarbeit. Teamarbeit ist also ein „Muss“ in der psychiatrischen Arbeit.

Zum Verständnis der Wichtigkeit gut funktionierender Teamstrukturen: Ein Team ist eine besondere Form der Arbeitsorganisation, bei der es zwingenderweise um Zusammenarbeit geht. Die Annahme, ein Team sei ein freiwilliger Zusammenschluss von Personen mit gleichen Rechten und Pflichten und gegenseitiger Sympathie, ist irreführend. Bei einem Team geht es immer um mehrere Personen, die sich einer gemeinsamen Aufgabe stellen wollen oder müssen.

Die Aufgabe lässt sich zwar aufteilen aber nicht unabhängig voneinander bearbeiten. Die gemeinsame Bearbeitung muss daher aufeinander abgestimmt sein.

Dörner/Plog schreiben in ihrem Buch „Irren ist menschlich“ zur Teamarbeit:

- Keiner kann die Wirklichkeit als Einzelner angemessen wahrnehmen oder verstehen.

- In dem, wie ich mich verstehe, ist enthalten, wie ich die Umwelt zu Teilen von mir gemacht habe.

- Den Zugang zu diesen Anteilen erhalte ich über das Gespräch mit anderen.

Diese Merksätze gelten selbstverständlich auch für den Patienten.

Weiter ...

- ... therapeutischer und diagnostischer Alleingang schließt andere „Wissende“ aus.

- ... dem Patienten steht somit das Wissen, das er braucht, um sich zu verstehen, nicht vollständig zur Verfügung.

- Teamarbeit macht es möglich, dass die unterschiedlichen Sichtweisen gleichberechtigt zusammen gesehen werden.

- Ein abgerundetes und differenzierteres Wahrnehmen und Handeln ist möglich.

- Nur mit Teamarbeit ist es daher möglich, dem Patienten die Unterstützung zukommen zu lassen, die er benötigt.

- Partikularinteressen einzelner Personen oder Berufsgruppen schaden daher nicht nur der Zusammenarbeit und der Arbeitszufriedenheit, sondern auch sehr dem Patientenwohl. 
3 Ärzte und Pflege: Feindliche Geschwister, oder der Weg zur Zusammenarbeit!

- Letzterem sind Arzt und Pflege gleichermaßen verpflichtet. Ein wertschätzender Umgang, die Anerkennung der jeweiligen Profession und deren Aufgaben und Belastungen sind daher die entscheidenden Elemente guter Zusammenarbeit.

\section{Literaturempfehlung}

Buddeberg C (Hrsg.) (2004) Psychosoziale Medizin. 3. Aufl. Springer Verlag Heidelberg

Dörner K, Plog U (2012) Irren ist menschlich. 21. Aufl. Psychiatrie Verlag Köln

Rothgangel S (2010) Kurzlehrbuch der Medizinischen Psychologie und Soziologie. 2. Aufl. Thieme Verlag Stuttgart 